\title{
ASSESSING DRIVER BEHAVIORAL ADAPTATION TO A RURAL INTERSECTION DRIVER SUPPORT SYSTEM
}

\author{
Michael Manser \& Janet Creaser \\ HumanFIRST Program, ITS Institute, University of Minnesota \\ Minneapolis, Minnesota, USA \\ Email: mikem@me.umn.edu
}

\begin{abstract}
Summary: Driver support systems have the potential to improve driving safety. However, most research only evaluates initial performance with the system and does not evaluate continued adaptation to the system to determine if the benefit continues or is negated by unintended use of the system. The efficacy of a previously evaluated rural intersection driver support system was examined in a simulated driving environment relative to system introduction, continued use, and potential positive transfer/carry over effects. Participants drove through a simulated rural intersection twelve times each day for a week with an intersection decision support system turned off during days one and five and turned on days two, three, and four. This experimental design allowed for an examination of the efficacy of the driver support system upon initial introduction, after continued use, and whether there were any carry-over effects. Results indicated drivers benefited from the rural intersection driver support system and that the benefit continued as exposure to the system continued. In addition, drivers continued to benefit from system use even after the system was no longer available. Results are discussed in terms of driver performance while using the system.
\end{abstract}

\section{INTRODUCTION}

Driver support systems (DSS) have the potential to reduce the cognitive load on drivers and free attentional resources that will allow them to focus better on primary driving tasks with the goal of reducing crashes on roadways. Research on driver performance when using a DSS typically only evaluates the immediate performance benefits of a system. However, behavioral adaptation over longer time periods can result in drivers engaging in unintended or unsafe behaviors while using a DSS, thus reducing safety overall and negating the initial safety benefit of the system. For example, initial evaluations of anti-lock brakes found that drivers reduced their braking distance significantly in poor weather conditions (Evans \& Gerrish, 1996), a finding that supported the utility of this particular DSS. However, allied research examining performance over longer periods of time found that drivers increased travel speeds (Rompe, Schindler, \& Wallrich, 1987) and reduced time headways to lead vehicles (Hoedemaeker \& Brookhuis,1998; Sagberg, Fosser, \& Saetermo, 1997) which potentially reduced a portion of the overall performance and safety benefits of the system. Drivers were apparently taking advantage of the system benefit to reduce their overall braking distance. While antilock brake systems are considered one of the many transportation safety success stories their true benefit was only revealed through longer-term evaluations that examined adaptation. Because adaptation to DSS, and vehicle-based technology in general, can significantly impact performance and safety, it is necessary to address adaptation when considering the veridical utility of these systems. 
Longer-term evaluations have the benefit of being able to determine whether drivers continually adapt to a DSS and whether such adaptation is associated with performance improvements or declines over time. The goal of this paper is to report the outcomes of an adaptation study using a DSS interface that had previously undergone rigorous testing both in simulation and in the field to examine its immediate effects on driver performance. The Cooperative Intersection Collision Avoidance System - Stop Sign Assist (CICAS-SSA) is an infrastructure-based DSS intended to help drivers make crossing decisions at a rural stop-controlled intersection (see Creaser et al., 2007; Creaser et al., 2010; Rakauskas et al., 2009). One limitation of previous research examining the utility of the CICAS-SSA is the lack of effort dedicated to addressing driver adaptation. This was not due to a lack of oversight on behalf of researchers but, instead, there was a need to first determine if the CICAS-SSA supported driver performance before committing further resources to an evaluation of adaptation. This is also a main reason why many studies do not examine adaptation over longer time periods. The current study aimed to examine immediate and short-term adaptation to the CICAS-SSA as well as post-use transfer effects over several days of use in a driving simulator. Based on previous research, it was hypothesized that performance using the CICAS-SSA would improve during the immediate adaptation period. Performance was also expected to remain consistent or improve across increasingly higher rates of exposure to the system due to the continued utility of the DSS across the short-term adaptation period. It was also expected that performance would return to preCICAS-SSA levels during the transfer period. Finally, this study demonstrates a method that allows the examination of immediate system performance, which a typical study collects, while also collecting information on short-term adaptation and transfer effects.

\section{EXPERIMENTAL METHODOLOGY}

\section{Participants}

Participants in this experiment were 9 females $\left(\mathrm{M}_{\text {age }}=33.2\right.$ years; $\left.\mathrm{SD}=18.8\right)$ and 12 males $\left(\mathrm{M}_{\mathrm{age}}=41.5\right.$ years; $\left.\mathrm{SD}=17.1\right)$ between 19 and 76 years of age $(\mathrm{N}=21)$ recruited from nearby rural communities. All participants possessed a valid Minnesota or Wisconsin driver's license for a minimum of four years, possessed a minimum of 20/40 vision or vision that was corrected to 20/40, had no physical or cognitive limitations that may have negatively influenced driving performance, and had no prior exposure to vehicle or infrastructure-based collision avoidance systems or driver support systems.

\section{Apparatus}

Driving Environment Simulator. The study was conducted using the HumanFIRST Program's driving environment simulator (Oktal; AutoSim) that consisted of a complete 2000 Saturn SC2 vehicle with realistic operational controls and instrumentation and a high-resolution visual scene (1.96 arc minutes per pixel) projected to a five-channel 210-degree forward field-of-view screen. Auditory and haptic feedback was provided by a 3D surround audio system, subwoofer, car body vibration system, and a three-axis electric motion system (roll, pitch, z-axis) system.

Test Intersection. To enhance the ability to identify behaviors representative of those that exist for drivers at a rural intersection, an exact replica of an intersection (located at Trunk Highway 
52 and County State Aid Highway 9 in southern Minnesota) was created in the driving environment simulator (see Figure 1). The simulator's traffic generation tool employed an algorithm that produced traffic streams based on the distribution and probability of gaps observed at the real intersection. Traffic patterns for each trial were unique to reduce confounds associated with repeated patterns. Simulated weather conditions included a partly cloudy sky with a slight haze in the distance to hide any 'popping' of vehicles on the horizon. Road conditions were dry to simulate those found normally at the intersection.

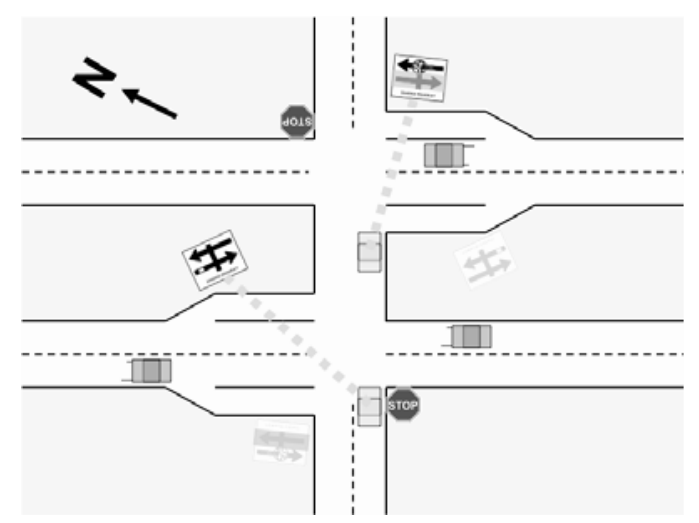

Figure 1. Diagram of the rural test intersection and locations of the CICAS-SSA interfaces at the intersection

CICAS-SSA. The CICAS-SSA presents an overview of the highway and the direction of vehicle traffic in the lanes. Icons (see Figure 2) indicate when traffic is detected near the intersection in each set of lanes; where "near lanes" indicate traffic traveling from left to right relative to the driver, while "far lanes" indicate traffic traveling from right to left in the far lanes of traffic relative to the driver. Creaser et al. (2010) provides a complete description of the CICAS-SSA.

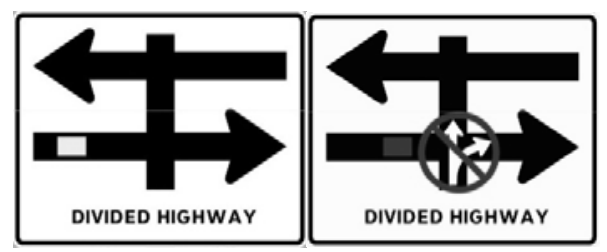

Figure 2. Two states of the CICAS-SSA sign presented to drivers on a minor road at a rural intersection

\section{Procedures}

Participants were introduced to the study, completed the informed consent process, and were provided with a brief description of the CICAS-SSA operation. Participants completed three practice drives without the CICAS-SSA present to become familiar with the simulator and the test intersection. Participants completed 12 experimental trials, in which they were instructed to start their vehicle, drive to the intersection, and then cross, turn left, or turn right as they would normally in actual driving situations. Turn maneuvers were counterbalanced between participants to avoid potential confounding due to order effects. Participants then repeated this pattern on Days 2-5 with the exception that a new counterbalancing order within and between 
participants was implemented each day to further reduce the potential for an order effect confound. The CICAS-SSA was deactivated for Days 1 and 5 and activated for Days 2, 3 and 4.

\section{Experimental Design}

Time (Days 1, 2, 3, 4, and 5) was the independent variable. Dependent variables included measures that evaluated crossing performance:

1. $80^{\text {th }}$ Percentile Rejected Gap - As described in the Microscopic Model analysis (Gorjestani et al., 2010) the $80^{\text {th }}$ percentile rejected gap can be used as a surrogate measure of the effect of the CICAS-SSA sign. The CICAS-SSA sign is intended to help drivers reject gaps that are smaller than 7.5 seconds, thus, we expected the $80^{\text {th }}$ percentile rejected gap to increase when drivers were presented with the CICAS-SSA. Gaps 15 seconds or greater were removed from the data prior to analyses due to the fact that these large gaps are generally accepted by all drivers.

2. Safety Margin - Time-to-contact (s) from when a participant's vehicle exited the mainline traffic flow. This is the time remaining before an approaching vehicle would have collided with a participant and is a measure of safety.

3. Movement Time Across Lanes - Time (s) to cross each set of lanes from entrance to exit. Slower movement times across a set of lanes can result in a reduced safety margin (not calculated for right turns or for far lanes during a left turn maneuver).

4. Wait Time - Time (s) participant waited at either the stop sign or in the median before crossing. This dependent variable may reflect time watching traffic, time watching and interpreting the CICAS-SSA information, and time making the decision to initiate a maneuver. Wait time was dependent on the gaps available to drivers.

Statistics. Performance variables were analyzed to examine the following differences:

1. Immediate Adaptation (Day 1 vs. Day 2): t-test, $p<0.05$

2. Short-term adaptation (Day 1 vs. Day 2 vs. Day 3): one-way ANOVA with Tukey HSD used for follow-up comparisons, $p<0.05$

3. Transfer (Day 1 vs. Day 5): t-test, $p<0.05$

\section{RESULTS}

Results of the evaluation of the 80th Percentile Rejected Gap found that for Days 1, 2, 3, 4, and 5 the 80th Percentile Rejected Gap was 6.3, 6.2, 5.9, 5.9, 5.9 respectively, which suggests drivers rejected smaller gap sizes when the CICAS-SSA was activated. Table 1 provides a summary of a cumulative frequency distribution of the rejected gap size for Days 1-5. Data indicate that at 7.5 seconds, the time at which the CICAS-SSA would warn of a gap drivers should not accept, the frequency of rejecting small gaps was lower for Day 1 compared to Days 2-5. 
Table 1. Rejected gap scores (percentages) for Days 1 through 5

\begin{tabular}{|c|c|c|c|c|c|c|c|c|c|c|c|c|c|}
\hline \multirow{2}{*}{ Day } & \multicolumn{13}{|c|}{ Rejected Gap Size (seconds) } \\
\hline & 1 & 2 & 3 & 4 & 5 & 6 & 7 & 7.5 & 8 & 9 & 10 & 11 & 12 \\
\hline 1 & 0.32 & 0.32 & 35.2 & 55.9 & 69.3 & 78.2 & 85.2 & 86.0 & 89.3 & 92.3 & 95.2 & 97.2 & 98.7 \\
\hline 2 & 0.18 & 0.24 & 32.9 & 54.9 & 69.1 & 79.2 & 87.2 & 88.3 & 92.0 & 94.7 & 97.1 & 98.1 & 99.1 \\
\hline 3 & 0.24 & 0.32 & 35.3 & 58.9 & 71.9 & 80.8 & 87.9 & 89.8 & 92.5 & 95.2 & 97.3 & 98.3 & 99.1 \\
\hline 4 & 0.14 & 0.42 & 36.0 & 59.6 & 70.9 & 80.8 & 87.6 & 89.2 & 92.4 & 94.9 & 96.9 & 98.1 & 99.2 \\
\hline 5 & 0.25 & 0.25 & 34.5 & 59.4 & 71.1 & 81.0 & 87.2 & 89.0 & 91.6 & 94.1 & 96.7 & 98.3 & 99.3 \\
\hline
\end{tabular}

Overall, the safety margin was significantly larger on Day 1 compared to Day 2, $t(19)=3.12$, $p=0.006$ (see Figure 3), indicating a decrease in safety margin upon immediate adaptation to the system. A significantly larger safety margin was also found for Day 1 compared to Day 5, $t(17)=2.93, p=0.009$, indicating that safety margin remained smaller during the transfer period.

Overall, movement times across lanes were significantly longer on Day 1 compared to Day 5, $t(17)=2.31, p=0.034$ (see Figure 3), indicating an effect of system use in the transfer period.
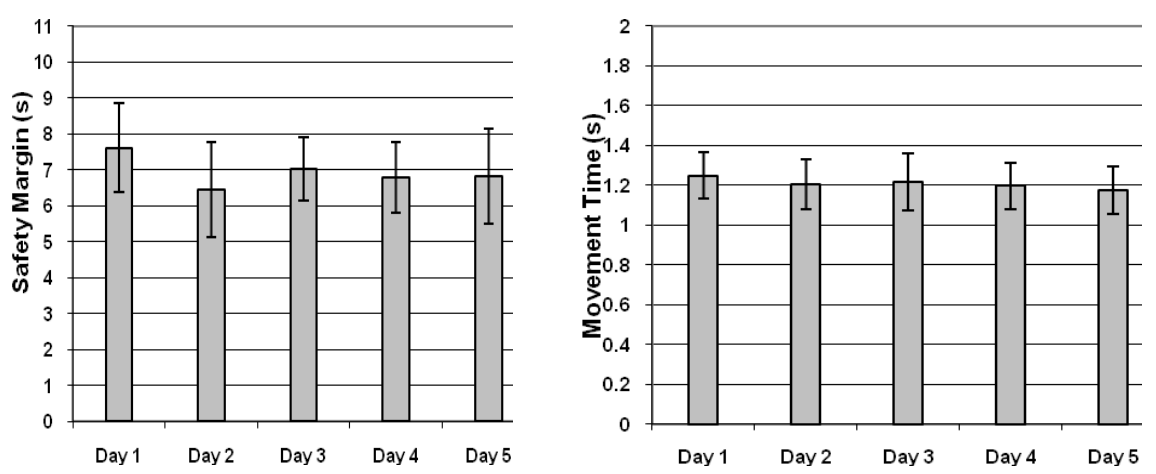

Figure 3. Safety margin and movement time means across days (with standard deviations)

Overall, wait times at the intersection were significantly longer on Day 1 compared to Day 5, $t(17)=3.00, p=0.008$ (see Figure 4), indicating shorter wait times occurred in the transfer period. Results also indicated a significant main effect of wait time across the short-term adaptation period (Days 2-4), $F(2,34)=4.94, p=0.019$. Post-hoc analysis indicated that Day 2 had significantly greater wait times compared to Day 4 (Tukey HSD, $p=0.039$ ).

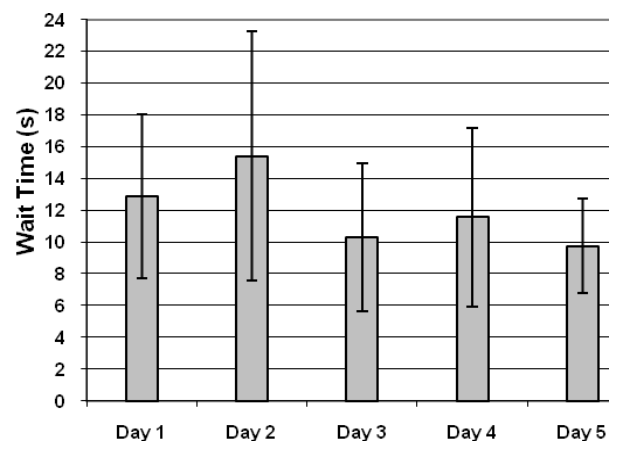

Figure 4. Wait Time means and standard deviations across days 


\section{DISCUSSION}

The primary goal of the current work was to determine if drivers adapted both immediately and in the short-term to a rural intersection-based driver support system based on their crossing performance. Additionally, transfer was examined by removing the system on the final day of the experiment. Overall, the immediate adaptation results of this study are similar to those found in the previous simulator studies of the CICAS-SSA, such as a smaller safety margin existing upon immediate interaction with the system (Creaser et al., 2010; Creaser et al., 2007). In the previous studies, the majority of drivers reported using the CICAS-SSA sign to help them with their crossing decisions, therefore, the shorter safety margins are a possible result of the increased processing time needed to examine the sign in conjunction with traffic to make a crossing decision. The smaller safety margins on Day 2 are not necessarily less safe because a $1 \mathrm{~s}$ buffer was built into the system's warning threshold to account for drivers to process the information. The actual drop in safety margin from Day 1 to Day 2 was $1.15 \mathrm{~s}$, indicating the buffer is reasonable.

In this study, significant short-term behavioral adaptation effects were observed in both the 80th percentile rejected gap profiles and the time drivers spent waiting at the intersection. The rejected gap profiles indicated that drivers rejected more gaps closer to the warning threshold with more system exposure. The wait times indicated that drivers initially had longer wait times upon first viewing the system but were significantly shorter by the third day of use. This suggests participants may have learned how the system worked and became able to respond quicker to the presented information when unsafe gaps were not detected. The reduction in wait times may also be due to drivers simply becoming more comfortable driving in the simulator over time. The lack of significant effects across system use days for safety margins and movement times suggest drivers did not change their behavior negatively with longer exposure to the system. Performance effects observed for immediate adaptation to system use remained constant with increased use.

Finally, transfer effects were seen for all performance variables with performance on Day 1 being significantly different than performance on Day 5. The rejected gap profile for Day 5 was consistent with system use days rather than with Day 1 . Safety margins were also larger on Day 1 compared to Day 5, but the data trend indicated that safety margins dropped more initially on Day 2 then stabilized at a value above the initial drop for subsequent system use days and the transfer period. Because the safety margin during transfer was similar to those on system use days, it suggests drivers became accustomed to crossing the intersection differently while using the system than they did on Day 1. Movement times were also longer on Day 1 versus Day 5. The observed transfer effects may be due to system use and/or increased familiarity with the simulator.

\section{CONCLUSIONS}

In general, the results indicate that using a longer-term study method is useful for obtaining both immediate performance results in addition to examining short or long-term adaptation and transfer effects. Overall, immediate performance in this study was consistent with previous studies and did not change with increased system use. However, there are some limitations to using simulation for an adaptation study. Because the driving took place in a simulated 
environment, it is possible the observed effects could be due in part to increasing familiarity or boredom associated with driving the simulator over a period of days. Ideally, an adaptation study for a driver support system would take place in the user's own vehicle to avoid changes due to learning that occurs during an experiment, but this is not always possible due to budget constraints. In this study, adequate practice drive times were used to help minimize familiarization effects. To help further illuminate the effects of adaptation, a field operation test is currently being conducted with the CICAS-SSA to examine adaptation effects in the real world while using the system.

\section{ACKNOWLEDGEMENTS}

The authors wish to acknowledge the support of the Intelligent Transportation Systems Institute, the HumanFIRST Program, and the Center for Excellence in Rural Safety for providing support to conduct this work. The authors also thank Peter Easterlund of the HumanFIRST Program for his efforts in building the driving environment simulator scenes and scenarios used in this work and Dan Drew for data collection.

\section{REFERENCES}

Creaser, J., Manser, M., Rakauskas, M., \& Donath, M. (2010). Sign comprehension, considering rotation and location, using random gap simulator for Cooperative Intersection Collision Avoidance System - Stop Sign Assist: CICAS-SSA Report \#4 (CTS 10-34). Minneapolis, MN: Center for Transportation Studies, University of Minnesota.

Creaser, J.I., Rakauskas, M.E., Ward, N.J., Laberge, J.C., \& Donath, M. (2007) Concept evaluation of intersection decision support (IDS) system interfaces to support drivers’ gap acceptance decisions at rural stop-controlled intersections. Transportation Research Part F, 10(3), 208-228.

Evans, L., \& Gerrish, P.H. (1996). Antilock brakes and risk of front and rear impact in twovehicle crashes. Accident Analysis and Prevention, 28(3), 315-323.

Gorjestani, A., Menon, A., Cheng, P., \& Shankwitz, C. (2010). Determination of the Alert and Warning Timing for the Cooperative Intersection Collision Avoidance System - Stop Sign Assist Using Macroscopic and Microscopic Data: CICAS-SSA Report \#1 (CTS 10-31). Minneapolis, MN: Center for Transportation Studies, University of Minnesota.

Hoedemaeker, M., \& Brookhuis, K.A. (1998). Behavioural adaptation to driving with an adaptive cruise control (ACC). Transportation Research Part F, 1, 95-106.

Rakauskas, M., Manser, M., Creaser, J. Graving, J., \& Donath, M. (2009). Validation Study: OnRoad Evaluation of the Stop Sign Assist Decision Support Sign: CICAS-SSA Report \#5 (CTS 10-35). Minneapolis, MN: Center for Transportation Studies, University of Minnesota.

Rompe, K., Schindler, A., \& Wallrich, M. (1987). Advantages of an anti-wheel-lock system (ABS) for the average driver in difficult driving situations. Proceedings of the 11th International Technical Conference on Experimental Safety Vehicles.

Sagberg, F., Fosser, S., \& Saetermo, I.F. (1997). An investigation of behavioural adaptation to airbags and antilock brakes among taxi drivers. Accident Analysis and Prevention, 29(3) 293-302. 